\title{
Unification of Surface Enhanced Raman Spectroscopy of Dyes Using One Pot Synthesized Stabilized Ag Nanoparticles
}

\author{
Vahideh Rezaie Kahkhaie ${ }^{a}$, Mohammad Hassan Yousefía, ${ }^{*}$, Seyyed Mohammad Reza \\ Darbani $^{b}$, Abolhassan Mobashery ${ }^{b}$ \\ aNano-Physics Center, Dept. of Physics, Malek Ashtar University of Technology, \\ Esfahan, Iran \\ ${ }^{b}$ Optics \& Laser Science and Technology Research Center, Malek Ashtar University of \\ Technology, Esfahan, Iran \\ *Corresponding Author's Email: mhyphd1@gmail.com
}

Received: Des. 18, 2018, Revised: Jul. 19, 2019, Accepted: Aug. 23, 2019, Available Online: Dec. 27, 2019

DOI: 10.29252/ijop.13.2.189

\begin{abstract}
Ag Nanoparticles (NPs) were synthesized using Lee-Meisel method under three different conditions in an oil bath. UV-Vis spectroscopy of the Ag NPs showed a Localized Surface Plasmon (LSP) band around $430 \mathrm{~nm}$, indicating Ag NPs had a size range around $40 \mathrm{~nm}$. To fabricate a surface Enhanced Raman Spectroscopy (SERS) substrate, LSP properties of Ag NPs was employed with the goal of detecting Rhodamine 6G dye. SERS spectrum was recorded by using 180 degrees, back-scatter Raman configuration in a custommade mount. The results showed that ideal Ag NPs agglomeration condition had been achieved by applying centrifuging process and due to this, adding $\mathrm{NaCl}$ salt to the SERS substrate was found to be unnecessary. The optimum rate of tri-sodium citrate versus silver nitrate and its influence on UV-Vis and SERS spectra was determined. It was understood that in order to obtain a uniform SERS intensity profile, employing a heater-stirrer instead of an oil bath alongside controlling the atmospheric condition and also drying the substrate in the Argon gas medium are the most necessary conditions for Ag NPs synthesize. The novelty point is obtained when SERS of R6G on a certain substrate, immediately after fabrication and after one month, were compared with a bare R6G dye substrate which, have revealed exceptional performance.
\end{abstract}

Keywords: SERS, Ag NPs, Lee-Meisel, LSP

\section{I.INTRODUCTION}

Nanomaterials are different from their bulk counterparts in various features like electronic, magnetic, stress and strain properties. Metal nanostructures especially transition metal nanoparticles have been excessively invested in the fields like nanocatalysts, biosensing and etc. [1]. Transition metals show exceptional properties. When a transition metal with specific condition is exposed to a certain wavelength of light, collective oscillation of electrons provokes localized high electromagnetic field zones called Localized Surface Plasmon Resonance (LSPR). Transition Noble metal nanostructures exhibit surface enhanced Raman scattering, throughout which, Raman scattering cross section of the molecules (analyte) adsorbed onto the metal nanostructure increases significantly. Since attenuation lines of silver exceed that of gold, and Plasmon resonance enhancement of it is greater, silver is preferred over gold. SPR band of silver could be set to all the visible wavelength of light and also, silver NPs cost much less than gold NPs. Disadvantageous to these features, mobility of $\mathrm{Ag}$ ions is fast and $\mathrm{Ag}$ ions oxidize easily [2\&3]. While these properties strongly affect the uniformity of SERS intensity and its 
reproducibility, the controllable synthesis of Ag NPs is still a challenge [4]. SERS is employed to detect different materials from biological to environmental analyts [5]. The Raman weak cross section limits its application, so the analyte molecules should be located in close proximity of plasmonic nanostructure [6] \& [7]. If nanoparticles have had a constant size distribution throughout the SERS sample and have been sufficiently close to each other, a strong interaction between the nanoparticles and analyte molecules would be gained [8]. Since there is no adequate and unique substrate, SERS is limited to each analyte where for each, a specific substrate has to be employed [9].

In this research, in order to unify the SERS intensity of dye molecules, Ag NPs were synthesized in three different conditions. Ag NP SERS substrate and Ag NP plus $\mathrm{NaCl}$ salt SERS substrate were fabricated to detect R6G dye Raman signal. Also, the effect of time and concentration of Ag NPs on Raman Intensity of R6G were investigated by employing UVVis spectroscopy and SERS.

\section{EXPERIMENTAL Procedure}

\section{A. Material and Methods}

$\mathrm{AgNO}_{3}$, tri sodium citrate, Ethanol, Acetone were provided from sigma Aldrich. All of the chemical reagents were used as received. Deionized water was used in all experiments. in the beginning, all of the lab glassware were washed with chromic acid, and then rinsed with deionized distilled water several times.

\section{B. Ag NP Synthesis}

Stabilized Ag NPs was synthesized using LeeMeisel method in an oil bath [10\&11]. An aqueous solution with $0.5 \mathrm{mM} \mathrm{AgNO}_{3}$ was heated while stirring in the oil bath. In order to have a constant temperature, it was controlled in the reaction medium using a laser thermometer. Humidity was controlled using a cooling system. After that, $0.5 \mathrm{mM}$ aqueous solution of tri sodium citrate, with a $99 \%$ purity and $294.10 \mathrm{~g} / \mathrm{M}$ molar mass was simultaneously stirred while heating and then it was combined with the boiling solution of silver nitrate at once. The reaction was continued at the boiling temperature for one hour with stirring alongside controlling humidity. At the end of reaction, it was allowed to cool to room temperature. At last, multiple centrifuging, and rinsing processes was followed.

Table 1. Nanoparticle and SERS Samples

\begin{tabular}{ccc}
\hline & $\begin{array}{c}\text { Sample } \\
\text { Numbers }\end{array}$ & Description \\
\hline & S.N. 1 & $\begin{array}{c}\text { Ag NPs synthesized in } \\
\text { oil bath and then } \\
\text { centrifuged }\end{array}$ \\
\cline { 2 - 3 } & S.N. 2 & T:A-R: 3 \\
\cline { 2 - 3 } Samples of & S.N. 3 & T:A-R: 4.5 \\
\cline { 2 - 3 } Nanoparticles & S.N. 4 & $\begin{array}{c}\text { Ag NPs synthesized in } \\
\text { oil bath, no } \\
\text { centrifuging followed }\end{array}$ \\
\cline { 2 - 3 } & S.N. 5 & $\begin{array}{c}\text { Ag NPs synthesized } \\
\text { using heater-stirrer, Ar } \\
\text { gas }\end{array}$ \\
\hline & S.S.1 & R6G+Ag NPs \\
\cline { 2 - 3 } & S.S. 2 & R6G+NaCl+Ag NPs \\
\cline { 2 - 3 } SERS Samples & S.S. 3 & R6G+S.N. 2 \\
\cline { 2 - 3 } & S.S. 4 & R6G+S.N. 3 \\
\cline { 2 - 3 } & S.S. 5 & R6G+S.N. 1 \\
\cline { 2 - 3 } & S.S. 6 & R6G+S.N. 4 \\
\cline { 2 - 3 } & S.S. 7 & R6G+S.N. 5 \\
\hline
\end{tabular}

\section{Fabrication of SERS Substrate and SERS Setup}

The glass slides were cut into $4 \mathrm{~cm}^{2}$ pieces and then washed with ethanol, acetone and distilled water, respectively. After that, SERS samples were prepared to investigate R6G SERS detection. Ag NPs were drop cast onto the glass substrate and it was allowed to dry in room condition. At last, R6G dye with a 0.1 $\mathrm{mM}$ concentration was deposited onto the glass piece. Fabrication of the S.S.2 was the same, except that, it carried with itself ratio of 1:1 Ag NP: $\mathrm{NaCl}$ salt.

Schematic SERS layout is shown in Fig. 1. At first, The second harmonic $\mathrm{CW}$ (Nd:YAG) laser with $20 \mathrm{~mW}$ pass through the band pass filter (FL532-10), and is split by dichroic beam separator Di02-R532. Next, the reflector element is sending the laser beam to the lens and then to the sample (it was held with sample holder) it enters the spectrometer. 
Scattered beam from the sample is collected using ICCD istar (HD334) and then it transmits through the band stop filter (NF53317), to enter the spectrometer (Ocean Optics HR4000CG-UV-NIR), which collects the sample traces.

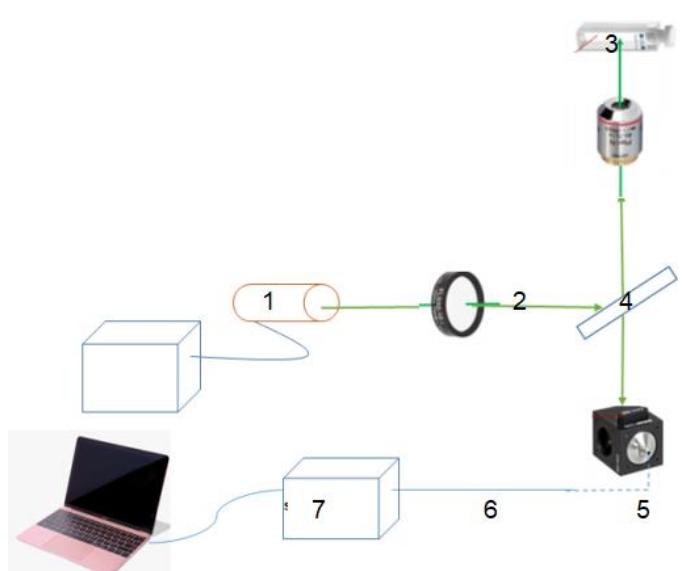

Fig. 1. SERS Schematic layout: 1 Laser, 2 Band pass filter 3 Sample, 4 Dichroic beam Separator, 5 Band stop filter, 6 Fiber, and 7 Spectrometer

\section{III.RESULTS AND DISCUSSION}

UV-Vis of S.N. 1 is shown in Fig. 2. In this figure, two shoulders were shown at $404 \mathrm{~nm}$ and $428 \mathrm{~nm}$ attributed to the Ag NP with 40$50 \mathrm{~nm}$ size range. We found this Particle size distribution similar to the experimental and simulation results which was obtained by Shen, et al. [4, 14-16], for silver and gold respectively. The UV-Vis and SEM shown in Fig. 3 proved this as the desired size of $\mathrm{Ag}$ NPs for SERS measurements, was proved by [4].

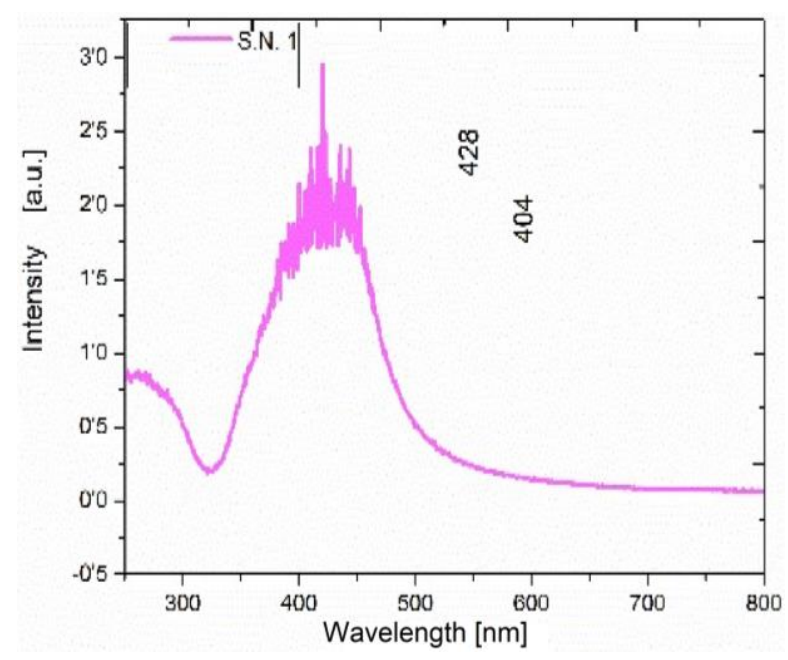

Fig. 2. UV-Vis absorption of sample S.N.1.

FE-SEM image of S.N. 1 shown in Fig. 4 presents spherical profile and homogeneous distribution for Ag NPs. It also determines a size range of 30 to $40 \mathrm{~nm}$ for $\mathrm{Ag} \mathrm{NPs}$ synthesized in the oil bath.

SERS signal of S.S.1 and S.S. 2 (R6G plus Ag NPs and R6G plus $\mathrm{NaCl}$ plus Ag NPs, respectively) are presented in Fig. 5. It is clear that absence of $\mathrm{NaCl}$ positively affected the Raman spectrum of R6G. Appropriate agglomeration produces a desired SERS intensity [17] which was obtained through several centrifuging processes.

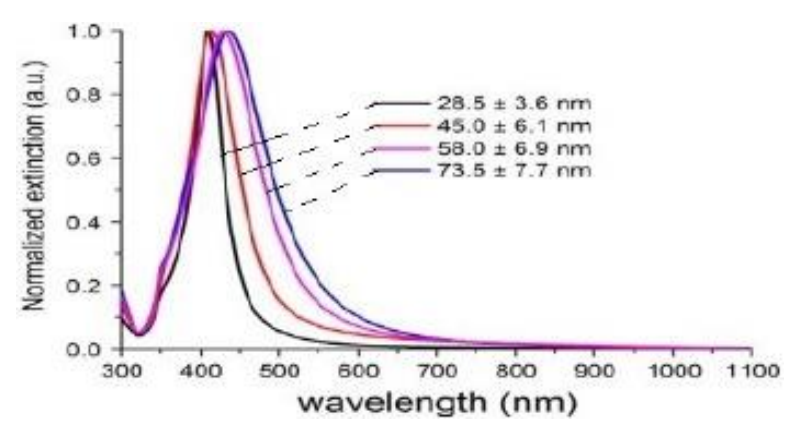

(a) 


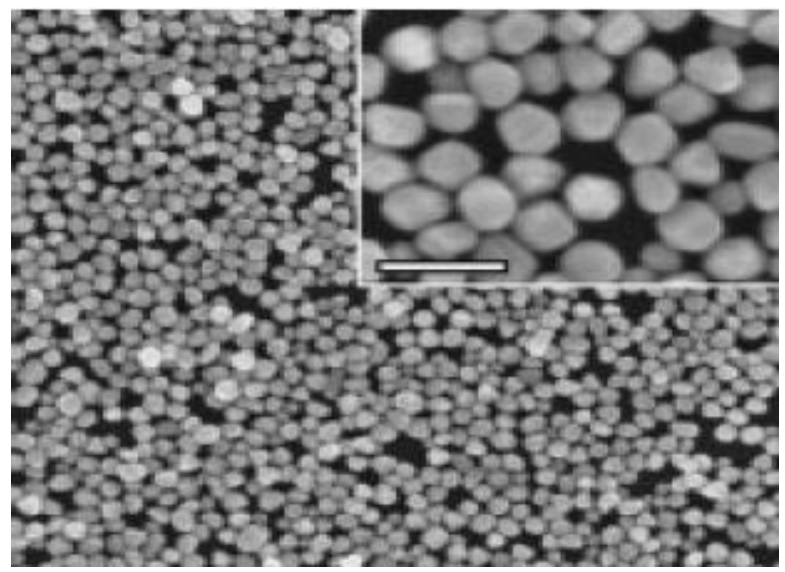

(b)

Fig. 3. (a) UV-Vis spectra and (b) SEM images of 28 to $75 \mathrm{~nm} \mathrm{Ag} \mathrm{NPs} \mathrm{[4].}$

Increased adsorption of dye pigments due to more vacant positions around Ag NPs have resulted in improved SERS signal in S.S. 1. Stronger fluorescent observed in the original graph (not shown here) is a proof for this conclusion.

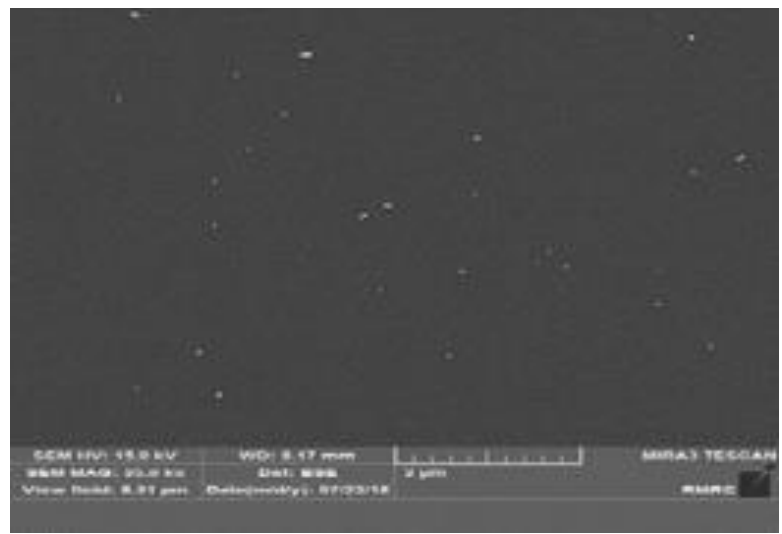

(a)

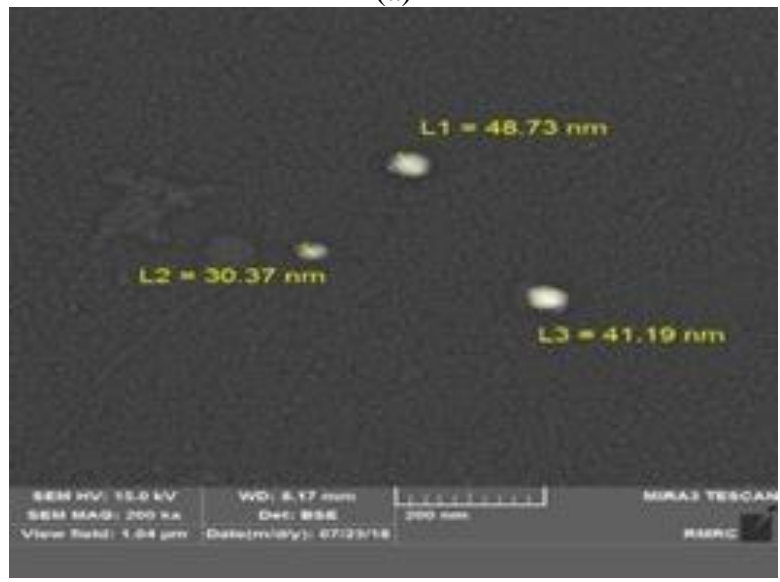

(b)

Fig. 4. (a) Image of Ag NPs size distribution and (b) The exact size measured by FE-SEM for synthesized Ag NP.
In S.S.2, SERS intensity dropped due to decrease in the appropriate hot sites and absorption of R6G molecules. This is caused by aggregation of R6G dye and big clusters of Ag NPs created by the addition of $\mathrm{NaCl}$ salt. Also we claim that, for the first time, the detection of R6G SERS was observed for peaks located at 354, 851, 979.03 and 1072.17, are shown in Table 2 and in Fig. 4.

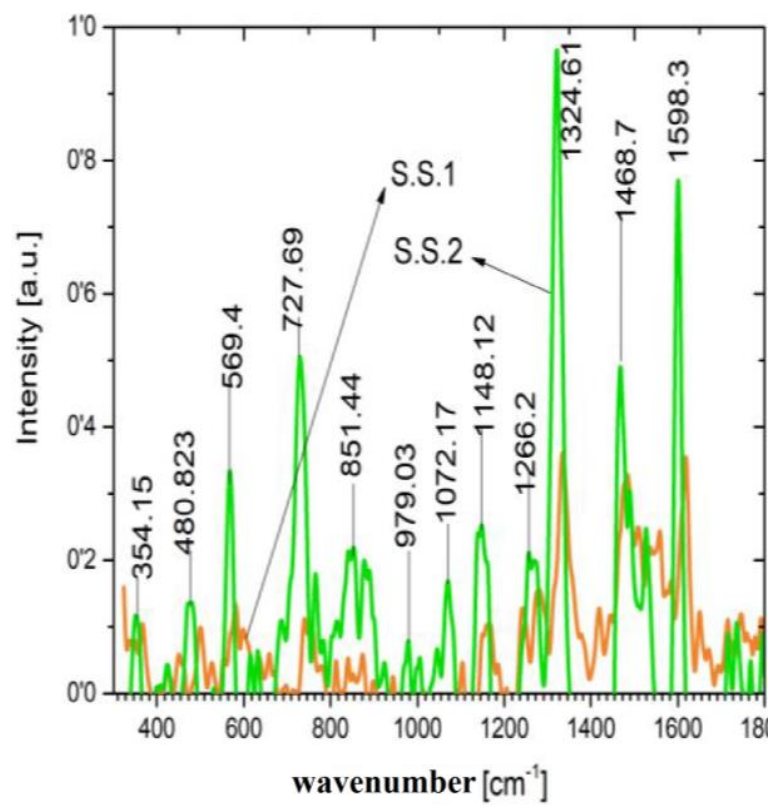

Fig. 5. SERS of S.S.1 and S.S.2.

Table. 2. Comparison of experimental and literature values of different vibrational modes of R6G.

\begin{tabular}{|c|c|c|}
\hline $\begin{array}{l}\text { Vibrational } \\
\text { modes of } \\
\text { functional } \\
\text { groups }\end{array}$ & $\begin{array}{c}\text { Literature } \\
\text { values }\left(\mathrm{cm}^{-1}\right) \\
{[18,19,20]}\end{array}$ & $\begin{array}{c}\text { Experimental } \\
\text { Values } \\
\left(\mathrm{cm}^{-1}\right)\end{array}$ \\
\hline $\begin{array}{l}D_{2 h}^{15} \text { symmetric } \\
\text { group frequency }\end{array}$ & - & 354 \\
\hline $\begin{array}{l}\text { C-H out of plan } \\
\text { bend }\end{array}$ & 785 [18] & 727 \\
\hline \multirow{3}{*}{$\begin{array}{l}\text { Ring breathing } \\
\text { vibration }\end{array}$} & - & 851 \\
\hline & - & 979.03 \\
\hline & - & 1072.17 \\
\hline $\begin{array}{l}D_{2 h}^{15} \text { symmetric } \\
\text { group frequency }\end{array}$ & 1186.9 [18] & 1148.12 \\
\hline $\begin{array}{l}\text { C-H out of plan } \\
\text { bend }\end{array}$ & $1303[18,19]$ & 1266 \\
\hline $\begin{array}{c}\text { Ring breathing } \\
\text { vibration }\end{array}$ & $1357[18,19]$ & 1324 \\
\hline \multirow{2}{*}{$\begin{array}{l}\text { Aromatic C-C } \\
\text { stretching }\end{array}$} & $1508[18,19]$ & 1468 \\
\hline & $1647[18,19]$ & 1598 \\
\hline
\end{tabular}




\section{IV.THE EFFECT OF TRI-SODIUM Citrate / $\mathrm{AgNO}_{3}$ Ratio}

Ag NP synthesis was repeated using different Tri-sodium citrate: $\mathrm{AgNO}_{3}$ Ratio (T: A-R) to provide for SERS substrate reproducibility and investigate the effect of tri sodium citrate on the nanoparticle size distribution. UV-Vis of S.N.2 and S.N.3, and SERS of S.S. 3 and S.S. 4 are presented in Fig. 6 and 7. Table 1 includes descriptions of the samples.

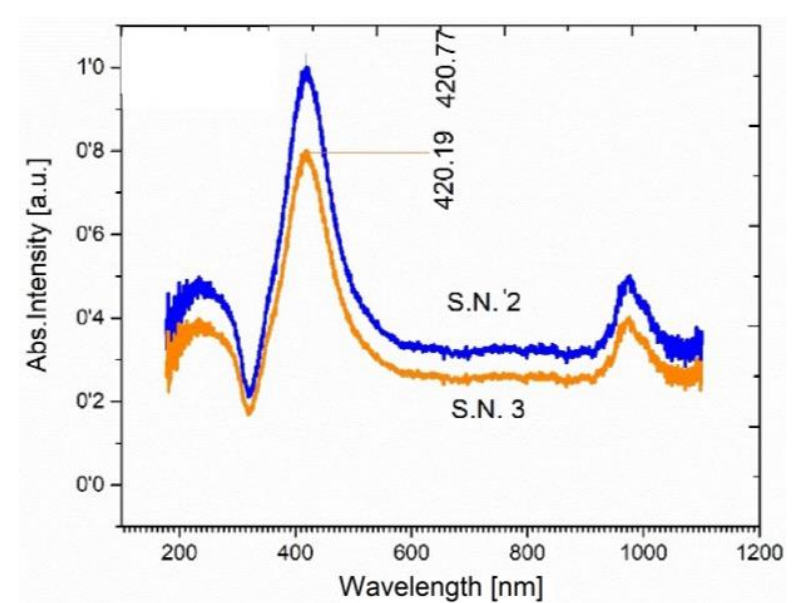

Fig. 6. Comparison between UV-Vis spectra of S. N.2 and S.N.3.

A slight blue shift is observed for the higher T: A-R (S.N. 3) in Fig. 6 (UV spectrum), which is caused by the decrease in the Ag NPs size. Increase in the number of tri sodium citrate molecules, limits the $\mathrm{Ag}^{+}$ions to attach to the Ag nuclei, so a smaller size is gained.

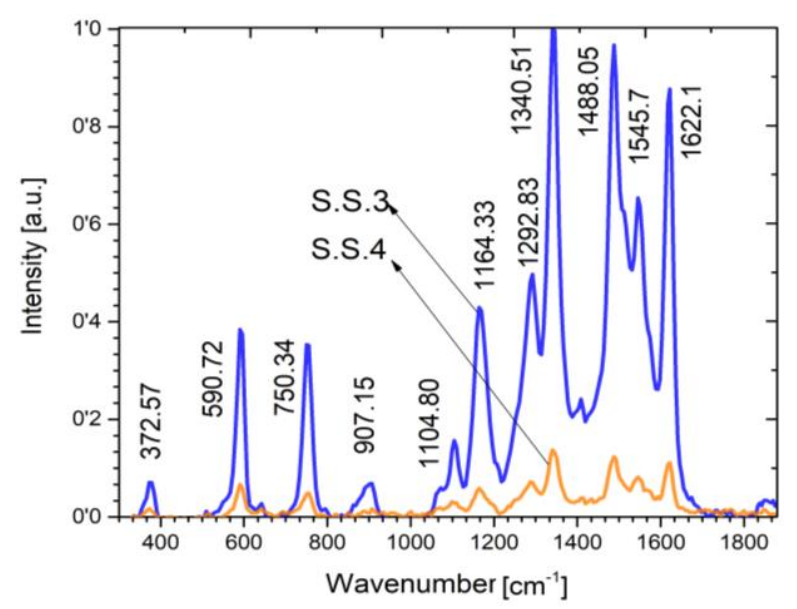

Fig. 7. Comparison of SERS spectra of S.S. 3 and S.S. 4.
The smaller Ag NPs in S.N. 2 could not compete with the bigger ones in S.N. 3 in terms of SERS spectra. An intensity ratio over 4 times is observed for S.N. 2. Comparison between Fig. 6 and 7 verify the dependence between UV-Vis and SERS spectra.

\section{The EfFect of Centrifuging AND RINSING}

S.N. 1 was synthesized the same way mentioned in the experimental section. But S.N.4 was processed without followed centrifuging.

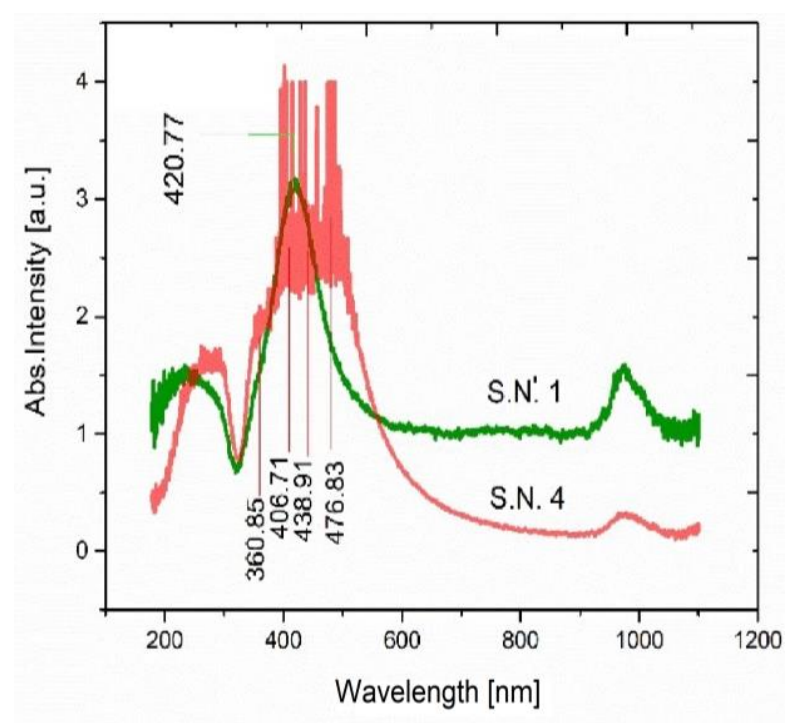

Fig. 8. UV-Vis spectra of S.N. 1 and S.N. 4.

UV-Vis spectra of these samples, is shown in Fig. 8 which presents a Gaussian spectrum, single plasmon peak and a smaller FWHM band for S.N.1. On the contrary S.N. 4 shows unwanted additional peaks at $360 \mathrm{~nm}, 406 \mathrm{~nm}$, and $476 \mathrm{~nm}$ which intervene with the plasmon band, hides it and broaden the FWHM band of the Ag NPs. Because band width of S.N. 1(80 $\mathrm{nm}$ ) and S.N. 5 overlap in similar intensities, the chance of size distribution being around 40 to $50 \mathrm{~nm}$ is high.

Figure 9 describes SERS of S.S.1 versus S.S.5. Size distribution difference between S.N. 1 and S.N. 4 presented itself through decrease in SERS intensity. So, SERS spectrum was affected by nanoparticle size distribution. 
Compared with UV-Vis analysis, it shows a smaller noise which proves that SERS is not as affected by the extra salts synthesized during reaction.

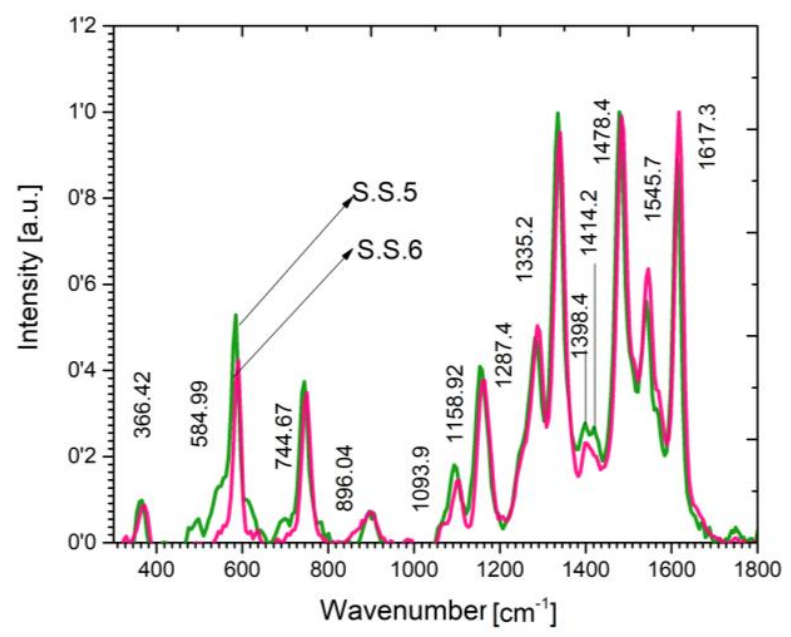

Fig. 9. SERS spectra of S.S. 5 and S.S. 6.

\section{VI.SERS SUBSTRATE OPTIMIZATION}

When an oil bath is employed in the fabrication of $\mathrm{Ag} \mathrm{NPs}$, un-uniform SERS spectrum is observed [12]. If medium is filled with oxygen around the reaction flask, the possibility of having a few oxidized Ag NPs and an inhomogeneous Ag NP colloid increase. This would cause lack in single size distribution and distorted adsorption of analyte molecules onto the Ag NPs and excites different localized surface Plasmon resonance modes which dampen the SERS signal [13].

To reach an optimum condition for Ag NPs synthesis, instead of the oil bath, the magnetic heater was used along with stirring to obtain positive results. Reaction atmosphere was deployed of oxygen by flowing argon gas with 100 mbar pressure. If $\mathrm{Ag}^{+}$is exposed to light, it shows great tendency to graft with oxygen ions. To avoid this effect, we isolated reaction flask with a thick aluminum foil, also creating a cavity that reflect the heat back into the flask.

Figure 10 shows UV-Vis spectrum of S.N. 5. Figure 11 shows SERS spectra of S.S. 7. It is seen that each point of SERS substrate acts as a very capable Raman enhancer. SERS intensities obtained shows a 0.72 to 1 ratio which is a promising for unification of SERS throughout samples.

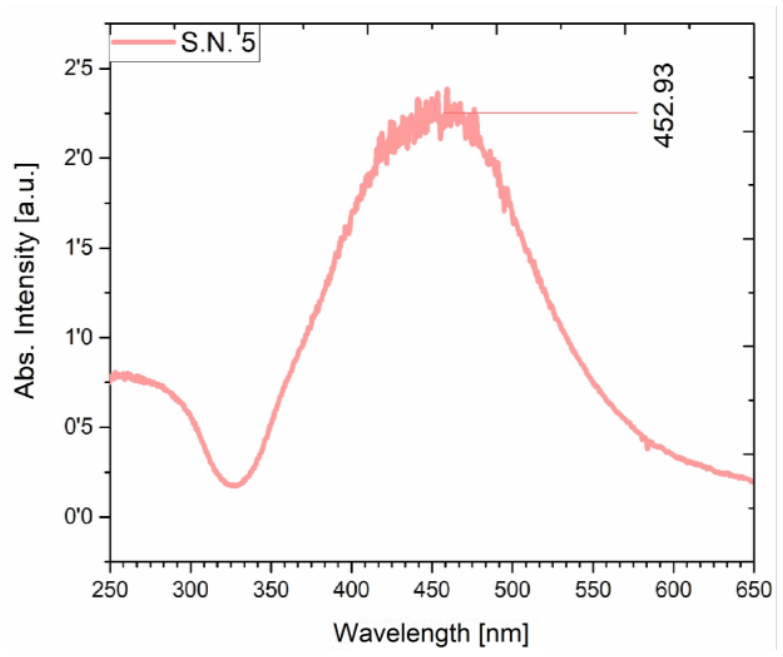

Fig. 10. UV-Vis spectrum of S.N. 5.

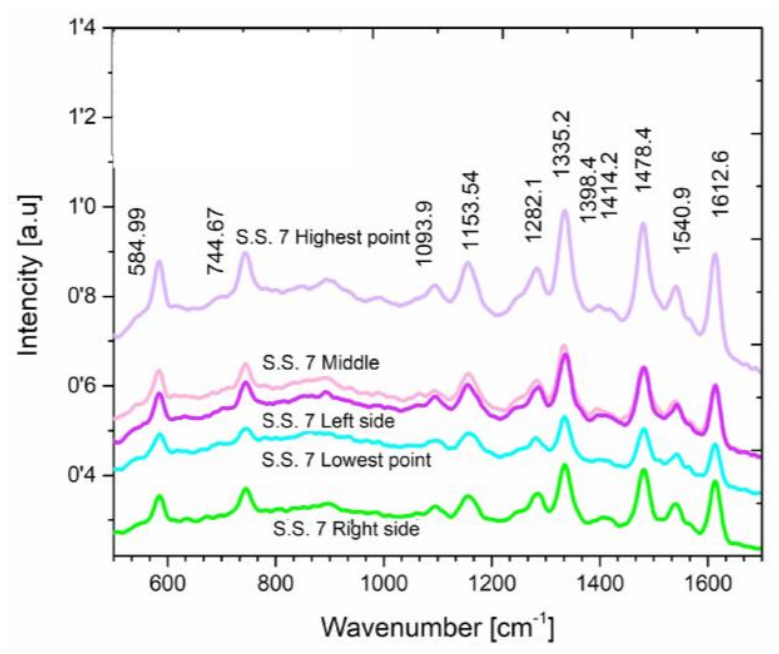

Fig. 11. SERS spectra at different positions of S.S. 7.

Considering the drop-cast method used to fabricate SERS substrate, these results qualifies our method of Ag NP synthesis and it creates a path for our future researches.

\section{DETERMINING THE Life TIME OF SERS SAMPLE}

With the goal of researching an optimum life time for our SERS samples were tested S.S. 7 sample immediately after fabrication and one month after. Also to compare the results, 
Raman spectrum of bare R6G is given. Fig. 12 shows SERS response of S.S. 7 compared with its older version and a bare R6G substrate. SERS without Ag NPs shows only perturbation. Raman spectrum of $\mathrm{R} 6 \mathrm{G}$ is difficult to detect because of its strong fluorescence. When SERS spectrum is collected right after substrate fabrication, it shows Raman peaks of R6G, not the background noise appeared in the case of bare R6G. Even after one month, SERS substrate was significantly capable of detecting the analyte.

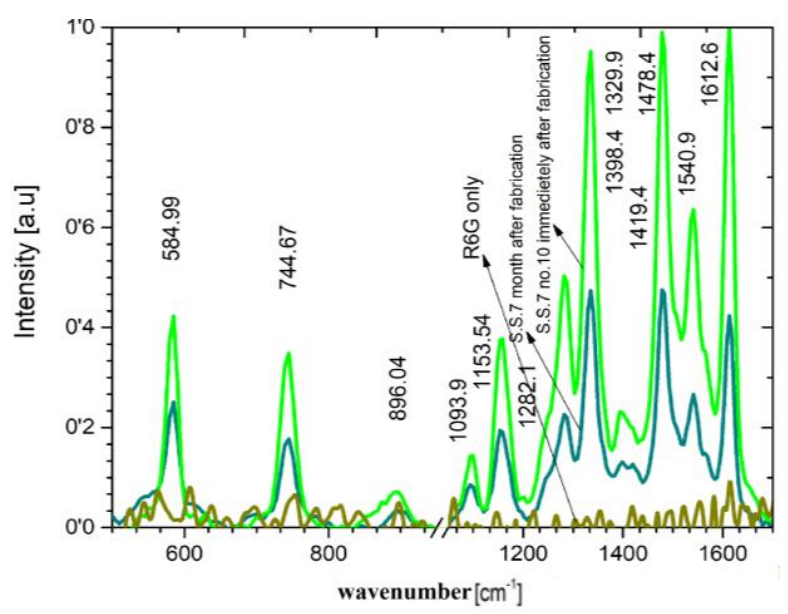

Fig. 12. Determination of life time of S.S. 7.

\section{VIII.CONCLUSION}

SERS sample with and without $\mathrm{NaCl}$ salt with ratio of $1: 1$ for the sample containing salt was fabricated.

The effect of Ag NPs size distribution, the synthesize root and the time interval on SERS and UV spectra was investigated.

The novelty and significant points were obtained as:

- The effect of size on UV-Vis are investigated which compared to the review literature, was found to be compatible with the FE-SEM and SERS observations.

- The SERS substrate without $\mathrm{NaCl}$ salt has given the outstanding results.
- The peaks at $354,851,979.03$ and 1072.17 were detected by SERS for the first time.

- Single size NPs, with a Gaussian profile for UV-Vis spectra was obtained, with an hour centrifuge process.

- The life time of SERS substrates was investigated. SERS signals gathered after one month showed exceptional compatibility.

- SERS intensities obtained 0.72 to 1 ratio uniformity throughout samples which is a promising for unification of SERS.

\section{REFERENCES}

[1] Xi. Hou, Xi. Zhang, Sh. Chen, H. Kang, and W. Tan, "Facile synthesis of $\mathrm{Ni} / \mathrm{Au}, \mathrm{Ni} / \mathrm{Ag}$ hybrid magnetic nanoparticles new active substrates for surface enhanced Raman scattering," Colloids and Surfaces A: Physicochem. Eng. Aspects, Vol. 403, pp. 148-154, 2012.

[2] J. Shen, Y. Zhu, Xi. Yang, J. Zong, and Ch. $\mathrm{Li}$, "Multifunctional Fe3O4@Ag/SiO2/Au Core-shell Microspheres as a Novel SERSActivity Label via Long-Range Plasmon Coupling," Langmuir, Vol. 29, pp. 690-695, 2013.

[3] V. Uzayisenga, Xi.-D. Lin, L.-M. Li, J.R. Anema, Zh.-L. Yang, Y.-F. Huang, H.-X. Lin, S.-B. Li, J.-F. Li, and Zh.-Q. Tian, "Synthesis, Characterization, and 3D-FDTD, Simulation of $\mathrm{Ag} @ \mathrm{SiO}_{2}$ Nanoparticles for Shell-Isolated Nanoparticle Enhanced Raman Spectroscopy," Langmuir, Vol. 28, pp. 9140-9146, 2012.

[4] Y. Wan, Zh. Guo, X. Jiang, K. Fang, X. Lu, Y. Zhang, and N. Gu, "Quasi-spherical silver nanoparticles: Aqueous synthesis and size control by the seed-mediated Lee-Meisel method," Adv. Colloid Interface Sci. Vol. 394, pp. 263-268, 2013.

[5] D. Philip, K.G. Gopchandran, C. Unni, and K.M. Nissamudeen, "Synthesis, characterization and SERS activity of $\mathrm{Au}-\mathrm{Ag}$ nanorods," Spectrochim. Acta A, Vol. 70, pp. 780-784, 2008.

[6] W. Meng, F. Hu, Xi. Jiang, and L. Lu, "Preparation of silver colloids with improved uniformity and stable surface-enhanced Raman scattering," Nanoscale Res. Lett. Vol. 10, pp. 34-42, 2015. 
[7] M. Shanthil, H. Fathima, and K. George Thomas, "Cost-Effective Plasmonic Platforms: Glass Capillaries Decorated with $\mathrm{Ag} @ \mathrm{SiO} 2$ Nanoparticles on Inner Walls as SERS Substrates," ACS Appl. Mater. Interfaces, Vol. 9, pp. 19470-19477, 2017.

[8] Qi Shao, F. Liao, and A. Ruotolo, "MagneticPolaron-Induced Enhancement of Surface Raman Scattering," Sci. Rep. Vol. 6, pp. 19025 (1-7), 2016.

[9] J. Li, S.K. Cushing, P. Zheng, T. Senty, F. Meng, A.D. Bristow, A. Manivannan, and N. $\mathrm{Wu}$, "Solar Hydrogen Generation by a CdS$\mathrm{Au}-\mathrm{TiO} 2$ Sandwich Nanorod Array Enhanced with Au Nanoparticle as Electron Relay and Plasmonic Photosensitizer," J. Am. Chem. Soc. Vol. 136, pp. 8438-8449, 2014.

[10]L. Polavarapu, A.L. Porta, S. M. Novikov, M. Coronado-Puchau , and L. M. Liz-Marzán, "Pen-on-Paper Approach Toward the Design of Universal Surface Enhanced Raman Scattering Substrates," Small, Vol. 10, pp. 3065-3071, 2014.

[11]Ch. Fan, "Tunable SERS effect of the silver rod and magnetite nanocomposite with different magnetic field and aspect ratios," J. Opt. Vol. 45, pp. 307-311, 2016.

[12] J. Kimling, M. Maier, B. Okenve, V. Kotaidis, H. Ballot, and A. Plech, "Turkevich Method for Gold Nanoparticle Synthesis Revisited," J. Phys. Chem. B, Vol. 110, pp. 15700-15707, 2006.

[13]M. Lismont, C. A. Páez, and L. Dreesen, "A one-step short-time synthesis of $\mathrm{Ag} @ \mathrm{SiO} 2$ core-shell nanoparticles," Adv. Colloid Interface Sci. Vol. 447, pp. 40-49, 2015.

[14] Y. Qin, Xi. Ji, J. Jing, H. Liu, H. Wu, and W. Yang, "Size control over spherical silver nanoparticles by ascorbic acid reduction," Colloids and Surfaces A: Physicochem. Eng. Aspects, Vol. 372, pp. 172-176, 2010.

[15] S. Hong and Xi. Li, "Optimal Size of Gold Nanoparticles for Surface-Enhanced Raman Spectroscopy under Different Conditions," J. Nanomater. Vol. 2013, pp. 790323 (1-9), 2013.

[16]K.G. Stamplecoskie and J.C. Scaiano, "Optimal Size of Silver Nanoparticles for Surface-Enhanced Raman Spectroscopy," J. Phys. Chem. C, Vol. 115, pp. 1403-1409, 2011.
[17]X. Wang, Y. Du, Q. Li, T. Wu, H. Hu, Y. Xu, H. Zhang, and Y. Pan, "Fabrication of uniform substrate based on silver nanoparticles decorated glycidyl methacrylate-ethylene dimethacrylate porous material for ultrasensitive surface-enhanced Raman scattering detection," J. Raman Spectros. Vol. 45, pp. 47-53, 2014.

[18] M. Wahadoszamen, A. Rahaman, N. M. R. Hoque, A. Talukder, K. M. Abedin, and A. F. M. Yusuf Haider, "Laser Raman Spectroscopy with Different Excitation Sources and Extension to Surface Enhanced Raman Spectroscopy," J. Spectrosc. Vol. 2015, pp. 895317 (1-8), 2015.

[19] A.M. Michaels, M. Nirmal, and L.E. Brus, "Surface Enhanced Raman Spectroscopy of Individual Rhodamine 6G Molecules on Large Ag Nanocrystals," J. Am. Chem. Soc. Vol. 121, pp. 9932-9939, 1999.

[20]C.P. León, "Vibrational Spectroscopy of Photosensitizer Dyes for Organic Solar Cells," Cuvillier, PhD Thesis, Dep. Physics, University of Bayreuth, Bayreuth, Lebanon, 2006.

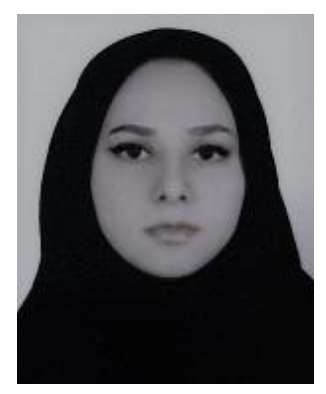

Vahideh Rezaie Kahkhaie completed her B.Sc. at Sistan and Baluchestan University, M.Sc. at Shahid Bahonar University of Kerman, Iran and $\mathrm{PhD}$ at Malek Ashtar University of Technology, Esfahan, Iran. Her main research works are in Nanostructures, especially in Plamonics. She is a member of Iran Nanotechnology and Physics Society of Iran. 


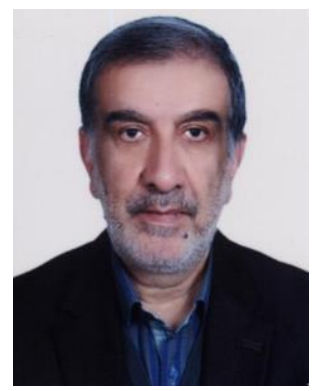

Mohammad Hassan Yousefi, an associate professor in Physics (Applied Electronics) at Malek Ashtar University of Technology completed his B.Sc., M.Sc. and Ph.D. studies at the Pune University, Poona, India. His main research works are in MEMS and NEMS, especially in QDs and Sensors. $\mathrm{He}$ is a member of Iran Nanotechnology, Physics Society of Iran, Optics and Photonics Society of Iran, and Vacuum Technology Society.

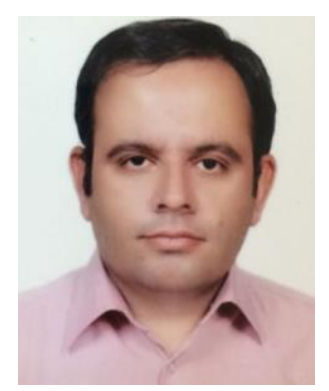

Seyyed Mohammad Reza Darbani, an assistant professor in Laser Optics at Malek Ashtar University of Technology completed his B.Sc. at Malek Ashtar University of Technology, M.Sc. and Ph.D. studies at the Isfahan University, Iran. His main research works are in Optic and Adaptive Optics. He is a member of Physics Society of Iran and Optics and Photonics Society of Iran.

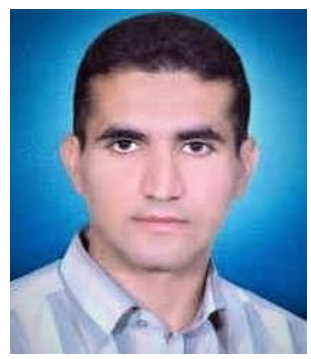

Abolhassan Mobashery, a researcher member in Laser Optics at Malek Ashtar University of Technology completed his B.Sc. at Shiraz University, M.Sc. and Ph.D. studies at the Isfahan University, Esfahan, Iran. His main research works are in Optic and Adaptive Optics. He is a member of Physics Society of Iran and Optics and Photonics Society of Iran. 
THIS PAGE IS INTENTIONALLY LEFT BLANK. 